\title{
Inflammasomes in Liver Fibrosis
}

\author{
Fernando Alegre, $\mathrm{PhD}^{1,2}$ Pablo Pelegrin, $\mathrm{PhD}^{3}$
1 Department of Pediatric Gastroenterology, University of California San Diego (UCSD), and Rady Children's Hospital, San Diego, California
2 Department of Pharmacology, University of Valencia and FISABIO- University Hospital Dr. Peset, School of Medicine, Valencia, Spain
${ }^{3}$ Director, Biomedical Research Institute of Murcia (IMIB-Arrixaca), CIBERehd, Clinical University Hospital Virgen de la Arrixaca,
Murcia, Spain

Ariel E. Feldstein, $\mathrm{MD}^{1}$

\begin{abstract}
Address for correspondence Ariel E. Feldstein, MD, Division of Pediatric Gastroenterology, Department of Pediatrics, Hepatology, and Nutrition UCSD, 3020 Children's Way, MC 5030, San Diego, CA 92103-8450 (e-mail: afeldstein@ucsd.edu).
\end{abstract}

Semin Liver Dis 2017;37:119-127.

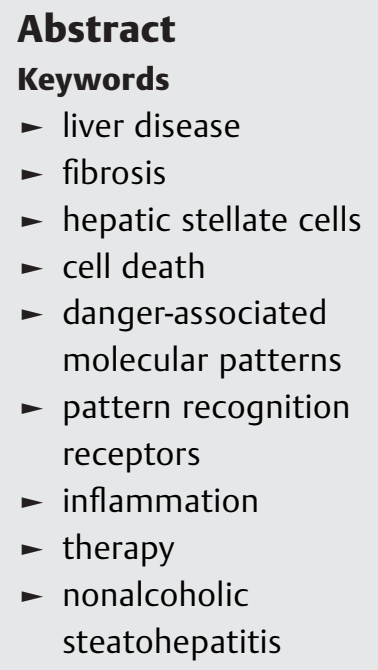

Cell death and inflammation are two central elements in the development of liver fibrosis. Inflammasomes are intracellular multiprotein complexes expressed in both hepatocytes and nonparenchymal cells in the liver that are key regulators of inflammation and cell fate. They respond to cellular danger signals by activating caspase 1 , releasing the proinflammatory cytokines IL-1 $\beta$ and IL- 18 , as well as initiating a novel pathway of programmed cell death termed "pyroptosis." These processes can initiate and perpetuate an abnormal wound-healing response with the principle cellular target being the activation of hepatic stellate cells. From the various inflammasomes, the NLRP3 inflammasome has been increasingly implicated in the pathogenesis of chronic inflammatory liver diseases, including nonalcoholic steatohepatitis, a disease process that is soaring and has evolved as a primary cause of liver fibrosis and need for liver transplantation. In this review, the authors highlight the growing evidence for both indirect and direct effects of inflammasomes in triggering liver fibrosis as well as potential novel targets for antifibrotic therapies.
Chronic liver disease represents a major cause of morbidity and mortality worldwide. ${ }^{1}$ Fibrosis is an intrinsic response to chronic persistent liver injury that results in a wound-healing process to mitigate the damage, but also can lead to scar formation. Liver fibrosis during its early stages is largely a silent process with no associated symptomatology making the diagnosis a significant clinical challenge relying mainly on an invasive liver biopsy. In addition, liver fibrosis can be stopped or reversed by removing the insult that triggered liver damage such as with the use of direct-acting antiviral agents directed to treat chronic hepatitis $\mathrm{C}$ virus (HCV) infection or bariatric surgery to induce significant and sustained weight loss in obese patients with nonalcoholic steatohepatitis (NASH). ${ }^{2}$ Unfortunately, in many instances liver fibrosis progresses over time and results in the development of cirrhosis, where the normal liver parenchyma is replaced by scar tissue. This results in severe disruption of the liver architecture and vascular distortion, and is associated with complications including portal hypertension, liver failure, and hepatocellular carcinoma, with liver transplantation as the only curative therapeutic option. Indeed, NASH has become increasingly common worldwide over the last decade, demonstrating the greatest increase as the cause of chronic liver disease among new liver transplant waitlist registrations-with a twofold increase in registrants in 2013 -to become the second leading etiology of liver disease. ${ }^{3,4}$ Dissecting the cellular and molecular mechanisms involved in the development and progression of fibrosis is an area of intense research interest and may result in the identification of novel antifibrotic approaches that would significantly aid in decreasing the global burden of liver disease.

\section{Fibrogenesis and Fibrosis}

\section{Key Cellular Players}

A central feature of fibrotic changes is the increased production and deposition of extracellular matrix proteins including collagen fibers, causing tissue scarring. Several cellular
Copyright @ 2017 by Thieme Medical Publishers, Inc., 333 Seventh Avenue, New York, NY 10001, USA Tel: +1(212) 584-4662.
DOI https://doi.org/ 10.1055/s-0037-1601350. ISSN 0272-8087. 
sources have been identified in both human and experimental studies that play an important role in the development of liver fibrosis, where the hepatic myofibroblasts are the most downstream cellular effectors of liver fibrosis, responsible for extracellular matrix deposition and scar formation, representing the activated hepatic stellate cell (HSC) as the main cell type involved in this process. Other cells include endogenous portal fibroblasts, myofibroblasts derived from liver parenchymal cells undergoing epithelial-mesenchymal transition, and to a lesser extent, bone marrow-derived fibrocytes. $^{5}$ Although the relative contribution of different sources of cells to the myofibroblast pool have been suggested to be determined by the different etiology of liver fibrosis, recent elegant fate tracing studies have revealed HSCs as the dominant fibrogenic effectors, independent of the etiology of the liver damage. ${ }^{6}$ Additionally, there are several other key cell types that play crucial roles during liver fibrosis development, which act upstream of HSCs by modulating their phenotype. In the context of chronic inflammatory-driven liver diseases such as NASH, two central additional cellular elements include the hepatocytes and innate immune cells, in particular macrophages and neutrophils. In the following section, we will focus on the importance of the crosstalk between these cell types that are tightly linked to the fibrogenic process.

\section{Cell Death and Sterile Inflammation}

Excessive hepatocyte cell death by apoptosis, necrosis, as well as other forms of cell death, in particular pyroptosis (discussed in the section below), represent strong triggers of HSC activation, fibrogenesis, and fibrosis. ${ }^{7}$ For instance, the attenuation of hepatocyte cell death also reduces HSC activation and scar formation in animal models of NASH and cholestasis, ${ }^{8,9}$ while hepatocyte-specific genetic disruption of the antiapoptotic member of the $\mathrm{Bcl}-2$ family $\mathrm{Bcl}-\mathrm{xL}$ results in hepatocyte apoptosis and liver fibrotic responses. In humans with $\mathrm{NASH}$, the extent of hepatocyte cell death strongly correlates with the severity of liver fibrosis. ${ }^{10}$ Various groups have explored the mechanisms linking hepatocyte cell death to fibrosis with early studies demonstrating that HSCs are capable of engulfing apoptotic bodies and this process stimulates their fibrogenic activity at least in part, via induction of the activation of reduced nicotinamide adenine dinucleotide phosphate oxidase 2, the phagocytic NADPH oxidase, in HSCs. ${ }^{11}$ Additionally, recent data have uncovered that dying hepatocytes release stressed signals also called danger signals, that trigger activation of a sterile (i.e., in the absence of infection) inflammatory response in cells of the innate immune system that induces further damage on neighboring hepatocytes and initiates a crosstalk with HSCs resulting in their activation. Moreover, these danger signals may also act directly on HSCs as modulators of their phenotype, as suggested by evidence that DNA released from apoptotic hepatocytes may act as an important mediator of HSC activation and differentiation resulting in the upregulation of transforming growth factor $\beta 1$ and collagen 1 messenger RNA in a process dependent on interaction with Toll-like receptor 9 in HSCs. $^{12}$ In the context of NASH, lipotoxicity, a process by which buildup of toxic lipids inside hepatocytes activates pathways of cell death, may result in the formation and release of extracellular vesicles that are efficiently internalized by HSCs, resulting in their activation in a process mediated by the shuttle of microRNAs and the suppression of peroxisome proliferator-activated receptor- $\gamma$ expression in HSCs. ${ }^{13}$ Putting together these studies support the novel concept that hepatocyte cell death with the release of extracellular danger signals and the activation of sterile inflammatory pathways can initiate an intrahepatic, self-perpetuating noxious loop central to the development of liver fibrosis. In the section below, we will introduce inflammasomes as novel key components of this loop.

\section{Inflammasomes and Downstream Effectors}

The inflammasomes are multiprotein complexes that control the activation of the proinflammatory caspase-1. Based in their structural domains, the main family of proteins described to form inflammasomes is the nucleotide-binding domain and leucine-rich repeat containing receptors (NLRs). Depending on the N-terminal domain, NLRs are divided into NOD-like receptor family, pyrin domain, containing protein 3 (NLRP) if it contains a pyrin domain (PYD) or NOD-like receptor family, CARD domain, containing protein 4 (NLRC) if it contains a caspase activation and recruitment domain (CARD).${ }^{14}$ Additionally, other proteins with PYD or CARD domains also form functional inflammasomes, such as interferon-inducible protein 16 , absent in melanoma-2, retinoic acid-inducible gene I and pyrin. ${ }^{15}$

The best characterized member of the NLR family is the NLRP3, which is a critical regulator of the innate immune response against a wide variety of triggers, including pathogens and sterile insults, suggesting that NLRP3 is a general sensor for tissue stress or damage. ${ }^{16}$ After activation, NLRP3 oligomerizes and recruits the adaptor protein apoptosis speck-like protein with a CARD domain (ASC), that initiates a prion-like oligomerization process with other ASC monomers, which is essential for the final structural conformation of the inflammasome. ${ }^{17,18}$ Finally, procaspase- 1 is recruited and activated within the inflammasome aggregate. ${ }^{19}$ Multiple intracellular signaling pathways have been proposed to activate the NLRP3 inflammasome. Nevertheless, although the rise of cytosolic $\mathrm{Ca}^{2+}$, the generation of mitochondrialderived reactive oxygen species (ROS), and the activity of lysosomal proteases are important to activate the NLRP3 inflammasome, ${ }^{20-22}$ the decrease of intracellular $\mathrm{K}^{+}$concentration appears to be a key common mechanism. ${ }^{23-25} \mathrm{Mi}-$ crobial pore-forming toxins induce a strong intracellular $\mathrm{K}^{+}$ depletion leading to NLRP3 activation; however, a decrease of intracellular $\mathrm{K}^{+}$also occurs after sterile damage sensing, that is after P2X7 receptor activation by extracellular adenosine triphosphate (ATP), ${ }^{26}$ after a decrease of extracellular osmolarity, ${ }^{27}$ or after activation by different metabolic lipids. ${ }^{9}$ However, molecules such as imiquimod and its derivatives do not deplete intracellular $\mathrm{K}^{+}$, but induce a robust NLRP3 inflammasome activation via the generation of mitochondrial-derived ROS. ${ }^{28}$ 


\section{IL-1 $\beta$ and Other Cytokines}

Interleukin- (IL-) $1 \beta$ is the most studied member of the IL-1 family of cytokines produced after inflammasome activation; it is an important proinflammatory cytokine with diverse biological activities and is implicated in multiple diseases. ${ }^{29}$ Pro-IL-1 $\beta$ protein lacks a signal peptide and does not follow the classical endoplasmic reticulum-Golgi route of secretion, ${ }^{30,31}$ instead, it requires its posttranslational processing by the protease caspase- 1 to release its mature form. ${ }^{32}$ Inflammasomes tightly control the activation of caspase- 1 , and hence the processing and secretion of bioactive IL- $1 \beta .{ }^{15}$ Mature IL- $1 \beta$ is released by unconventional protein secretion mechanisms that include microparticle shedding or release through pyroptotic hyperpermeable membranes (see below). ${ }^{33-36}$ IL-18 is another member of the IL- 1 family that is also synthesized as an inactive precursor lacking a classical signal peptide and requiring caspase- 1 posttranslational processing to release the mature form from the cell. ${ }^{29}$ Both IL-1 $\beta$ and IL-18 are classical cytokines driving inflammation after inflammasome activation by binding to their respective receptors. IL-1 $\beta$ through engagement of IL-1R type I and IL-18 through IL-18R, both activate NF-кB (nuclear factor kappa-light-chain-enhancer of activated $\mathrm{B}$ cells) signaling and induce a dedicated proinflammatory gene expression program in the target cell. ${ }^{29}$ Beyond caspase- 1 substrates pro-IL-1 $\beta$ and pro-IL-18, caspase- 1 also controls the unconventional release of other proteins from the cell that are not direct substrates of this protease, including IL$1 \alpha$ and a specific set of intracellular proteins known as the caspase-1 secretome. ${ }^{37-39}$ The secretome associated with caspase- 1 is mainly composed of proteins with an intracellular function, such as galectins or cystatins; however, under certain conditions these proteins could also demonstrate an extracellular function. Among them, we could highlight the nuclear protein high-mobility group box 1 (HMGB1), a protein with histone-binding properties that controls gene expression in the nucleus. ${ }^{37,38}$ The extracellular presence of these proteins is sensed as danger signals by the immune system because they are not in their right place": For example, extracellular HMGB1 engages the advanced glycation end-product-specific receptor in conjunction with Toll-like receptors (TLR) to induce an inflammatory response. ${ }^{40}$ So inflammasome signaling goes beyond the release of proinflammatory IL-1 $1 \beta$ to other cytokines and intracellular proteins that amplify the danger signaling beyond the initial injury site.

\section{Pyroptosis}

Activation of caspase- 1 by inflammasomes as well as activation of caspase-4, and its mouse orthologue caspase- 11 , by intracellular lipopolysaccharide induces a novel programmed type of cell death characterized by cell swelling, hyperpermeabilization of the plasma membrane, rapid cell lysis, and the consecutive release of cytoplasmic content. This type of cell death, termed "pyroptosis," is intrinsically proinflammatory because it is associated with the release of IL-1 $\beta$ and other intracellular content. ${ }^{41}$ Pyroptosis was initially characterized in macrophages infected with Salmonella, a stimulus-inducing caspase- 1 activation via the NLRC4 inflammasome. Later, it was described that pyroptotic cell death is driven by any pathway activating either caspase- 1 or 4. ${ }^{41-43}$ The molecular mechanism involved in pyroptosis has been recently described as dependent on gasdermin-D cleavage by these caspases. ${ }^{35,44,45}$ After caspase processing of gasdermin-D, its $\mathrm{N}$-terminal peptide fragment binds to the inner leaflet lipids of the plasma membrane and oligomerizes within the membrane to form pores with an internal diameter of 10 to $15 \mathrm{~nm} .^{35,46,47}$ Gasdermin-D pores are the executors of pyroptosis, and by size they could allow the release of mature IL-1 $\beta$; however, the specific permeability of gasdermin-D pores is not actually known. Furthermore, the gasdermin-D N-terminal fragment is released from pyroptotic cells into the extracellular milieu, where it exhibits bactericidal activity acting as an antimicrobial peptide. ${ }^{35}$ Gasdermin-D N-terminal does not disrupt the plasma membrane of viable mammalian cells from the outside due to its lipid-binding specificity, and therefore does not harm neighboring cells. ${ }^{35}$ The gasdermin family of proteins is composed of four members in the human genome that codify for gasdermin-A to D; although gasdermin-A, B, and C lack a conserved caspase cleavage site, the expression of their $\mathrm{N}$-terminal fragment is able to induce pyroptosis. Therefore, pyroptosis could be renamed as the specific cell death triggered by gasdermin pores, independently of the protease that could cleave gasdermin, and not as a specific type of cell death driven exclusively by caspase- 1 or $4 .{ }^{47}$

\section{Extracellular Inflammasomes}

Pyroptosis also induces the release of large protein complexes; this observation was initially described by Gabel's group. They found in the supernatants of hypotonic-treated macrophages a precipitable fraction of macromolecules. ${ }^{48}$ In fact, inflammasome oligomers, mainly formed by the adaptor protein ASC, are released from the macrophages upon caspase- 1 activation. ${ }^{49,50}$ The release of inflammasome particles is dependent on pyroptotic cell death and is a mechanism to spread inflammasome signaling to adjacent cells. Extracellular inflammasomes activate procaspase- 1 in cell-free environments because high concentrations of procaspase- 1 are present in the extracellular milieu. Also, extracellular inflammasomes are internalized by macrophages where they could activate caspase- $1 .{ }^{49,50}$ In vivo, a fraction of the formed inflammasomes is found in the interstitial fluid and macrophages release inflammasomes in the lymph node after viral infection. ${ }^{51,52}$ In different human pathologies, circulating inflammasome particles are found in the serum of cryopyrinassociated periodic syndromes (CAPS) during an inflammatory flare and in the bronchioalveolar lavage of chronic obstructive pulmonary disease patients. ${ }^{49,50}$ This new concept of inflammasome propagation opens new avenues to spread not only inflammation, but also pyroptosis from cell to cell.

\section{The Role of Inflammasomes as Novel Modulators of Liver Fibrosis}

Growing evidence supports a central role of inflammasomes and their downstream effectors in liver fibrosis development (-Fig. 1). The expression levels of inflammasomes increase 


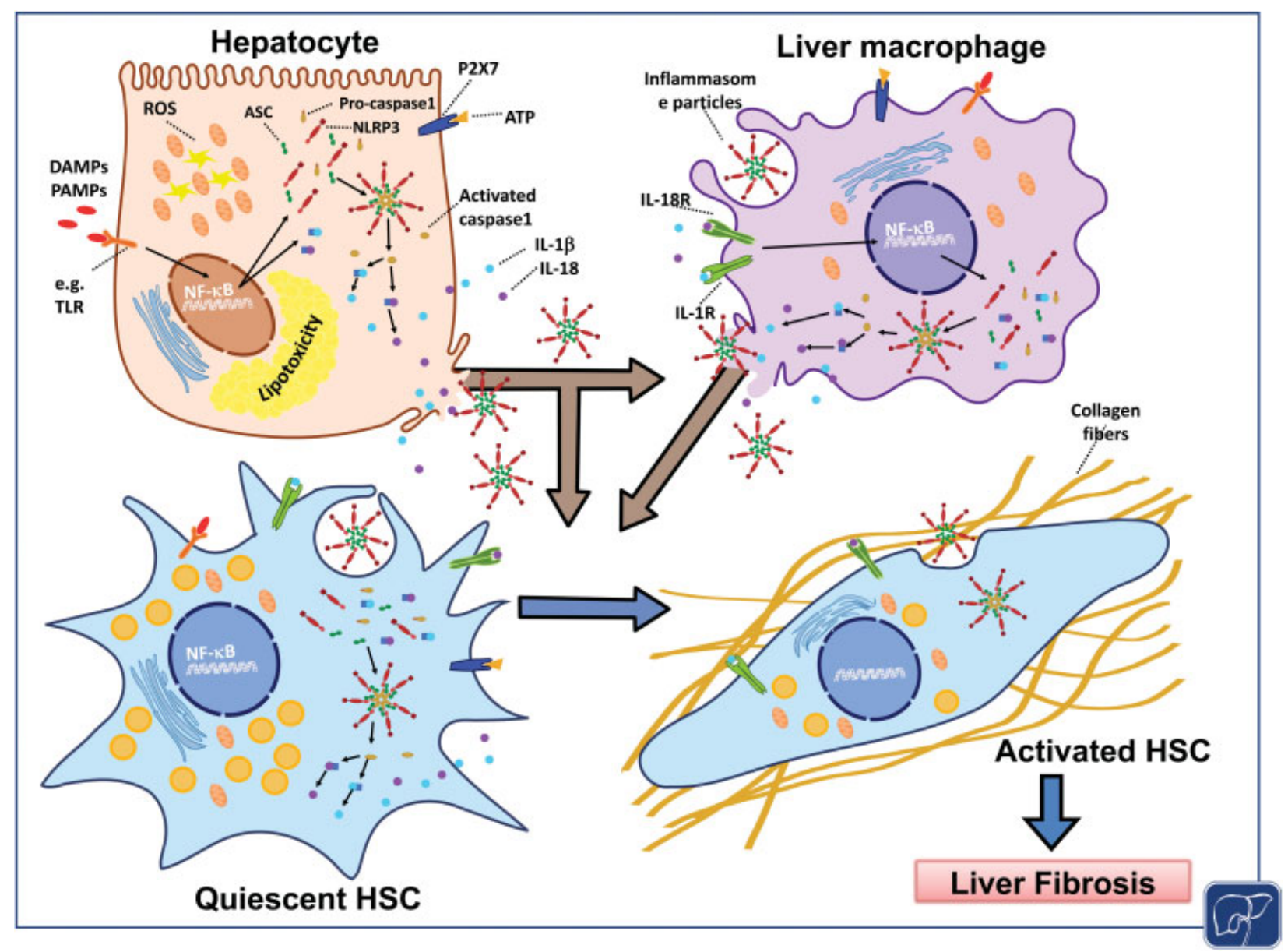

Fig. 1 The Role of the NLRP3 Inflammasome in Liver Fibrosis. Hepatocyte injury, induced by various deleterious agents, results in the release of damage-associated molecular patterns (DAMPs) and pathogen-associated molecular patterns, which could trigger NF-KB-mediated upregulation of NLRP3 inflammasome components. The presence of other cellular insults like ROS, oxidized mitochondrial DNA, lipid overloading, and/or P2X7 pathway (with $\mathrm{K}^{+}$efflux) may induce the activation of the NLRP3 inflammasome, thereby promoting hepatocyte pyroptotic cell death and releasing additional danger signals like IL-1ß/IL-18 or inflammasome particles. These events could take place likewise in nonparenchymal cells, like HSCs and liver macrophages, giving rise to an important crosstalk between all liver cellular types for the onset and progression of liver fibrosis. The cytokines and DAMPs generated by inflammasome activation will bind to receptors located on the HSCs and induce the upregulation of fibrotic markers, resulting in their activation that leads to liver fibrosis. ASC, adaptor protein apoptosis speck-like protein with a CARD domain; ATP, adenosine triphosphate; DAMPS, damage-associated molecular patterns; HSC, hepatic stellate cell; IL, interleukin; NF-KB, nuclear factor kappa-light-chain-enhancer of activated B cells; PAMPS, pathogen-associated molecular patterns; ROS, reactive oxygen species; TLR, Toll-like receptor.

during experimental liver fibrosis including NLRP-1, NLRP-3, and AIM2 and are prominently expressed in Kupffer cells and moderately expressed in HSCs. ${ }^{53}$ Isolated persistent activation of the NLRP3 inflammasome in mice results in severe liver inflammation, fibrosis, and hepatocyte pyroptotic cell death. ${ }^{54}$ In murine models of NASH, NLRP3 activation is required for the fibrotic response, suggesting that targeting this complex may be a rational strategy to block or reverse the development of fibrotic NASH. ${ }^{55}$ Among the various cytokines participating in chronic hepatic inflammation, IL-1 $\beta$ has been the one most extensively studied in liver fibrotic diseases, ${ }^{56}$ as well as in various others organs such as the lung ${ }^{57,58}$ and kidney. ${ }^{59}$ In vitro studies have shown that IL-1 $\beta$ promotes the proliferation and transdifferentiation of $\mathrm{HSCs}^{60,61}$ with a substantial increase in levels of their fibrogenic markers, including metalloproteinases, ${ }^{62,63}$ tissue inhibitor of metalloproteinases- 1 , collagen- $1 \alpha 1$, and trans- forming growth factor- $\beta$ (TGF- $\beta$ ), and a decrease in the expression of Bambi (a negative regulator of TGF- $\beta$ signaling). ${ }^{64}$ In vivo studies using global IL-1 $1 \beta$ or IL-1 receptor type I (IL-1RI) knockout mice have demonstrated that the IL-1 pathway deficiency protects against the progression of steatosis to steatohepatitis and liver fibrosis in both human-disease relevant murine models of NASH and alcoholic steatohepatitis. ${ }^{65}$ Similarly, these mice have also shown to be protective against various commonly used experimental models of liver fibrosis including the intraperitoneal injection of thioacetamide and bile duct ligation (BDL). ${ }^{66,67}$ Furthermore, IL1-R antagonist (IL-1Ra) knockout mice fed with atherogenic diet developed severe NASH and fibrosis with high levels of IL- $1 \beta$ and TGF- $\beta$ in the liver. ${ }^{56}$ Thus, significant evidence point toward the IL-1 pathway as an important mediator of the transition from liver injury to the onset of liver fibrogenesis and fibrosis. 
Similar to the findings using global IL-1 deficient mice, studies using mice genetically deficient in any of the three components of the NLRP3 Inflammasome: caspase 1, NLRP3, or ASC have also demonstrated a protective effect on fibrosis development. Indeed, global caspase-1 knockout mice are also protected from high-fat-diet-induced hepatic inflammation, and fibrogenesis, ${ }^{68}$ while in a model associated with severe fibrotic NASH, caspase-1 deficiency also protects from fibrosis development. ${ }^{69}$ In this study, the selective depletion of Kupffer cells potently suppressed methionine and choline deficiency-induced caspase- 1 activation and consequent fibrosis development. Wild-type mice on a choline-deficient, amino acid-defned (CDAA) diet showed increased hepatic caspase- 1 activity and liver fibrosis that were not observed in TLR2 knockout mice. ${ }^{70}$ More recently, using both global NLRP3 deficient mice and transgenic mice with constitutively global activation of NLRP3 we found that the former are protected from CDAA-induced NASH and fibrosis while the latter showed exacerbation of liver injury with a fibrotic phenotype when placed on a short-term CDAA feeding. ${ }^{55}$ The limitations of these studies include the use of global knockouts that does not allow to dissect the tissue / cell-specific role of inflammasome / IL-1 pathway activation as well as a lack of mechanistic information on whether the protection from liver fibrosis development is related to direct antifibrotic effects or indirect effects as a consequence of suppression of inflammation and cell death. In this regard, an early study from Mehal's group demonstrated that both primary murine HSCs as well as LX-2 cells, an immortalized human stellate cell line, express all components of the NLRP3 inflammasome and its activation using monosodium urate crystals, a potent signal two inflammasome activator, results in a phenotypic switch from quiescent to myofibroblast collagen-producing cells. ${ }^{71}$ Moreover, these changes were abrogated in HSCs isolated from ASC-deficient mice, suggesting the inflammasome activation in HSC might be sufficient to induce a fibrogenic response. To test this hypothesis in vivo, we recently developed HSC-specific NLRP3 knock-in (Nlrp3KI lecithin retinol acyltransferase Cre [CreLT]) mice by crossing mice expressing a mutational ortholog to D303N in human NLRP3, resulting in hyperactive NLRP3 on C57BL/6 background to C57BL/6-LRAT- CreLT mice allowing for efficient, selective expression of the NLRP3 mutation in HSCs. ${ }^{72}$ Nlrp3KI CreLT and wild-type littermates were maintained on a regular chow, low-fat diet for 6 and 9 months. We found that NLRP3 inflammasome activation in HSCs resulted in a marked increase in the number of $\alpha$ smooth muscle (a-SMA) positive cells, a key marker of activated HSCs with a perisinusoidal / pericellular distribution in the livers; this was associated with the spontaneous development of liver fibrosis. Thus, these data strongly support the concept that the NLRP3 inflammasome plays a direct role in liver fibrotic responses with significant implications related to the development of novel strategies for the treatment of liver fibrosis.

The association of NLRP3 inflammasome / IL-1 $\beta$ pathway to liver damage and fibrosis has been recently explored in human studies that revealed that the various components of the NLRP3 inflammasome as well as IL-1 $\beta$ levels in the livers are elevated in both patients with chronic $\mathrm{HCV}$ infection ${ }^{73}$ and those with $\mathrm{NASH},{ }^{55,74}$ whereas the levels correlated with markers of fibrogenesis and HSCs transdifferentiation into a myofibroblast phenotype, such as cysteine- and glycine-rich protein 2 (CRP2), $\alpha$-SMA, collagen-1 $\alpha 1$, and TIMP1. Other important activators of the NLRP3 inflammasome that contribute to chronic liver disease are ROS. In biopsies from patients with liver fibrosis, elevated levels of NLRP3 and NOX4 (a generator of ROS) in $\alpha$-SMA-positive cells were found. An increased NOX4 expression and ROS production in hepatic stellate cells result in the activation of the NLRP3 inflammasome and HSC activation with increased collagen production. Furthermore, treatment with antioxidants inhibited NLRP3 inflammasome activation and collagen synthesis in the liver. ${ }^{75}$ In addition, we have recently demonstrated that serum levels of caspase 1 activity strongly correlated with the severity of fibrosis on liver biopsy in a large cohort of patients with NASH. ${ }^{76}$ Translational studies to further explore the contribution of inflammasomes to liver fibrosis in patients with various chronic liver disorders are warranted.

\section{Therapeutic Implications}

Given the growing evidence for a role of NLRP3 inflammasome in liver injury and fibrosis, several studies have started to test the potential role of pharmacological modulation of this pathway as an antifibrotic therapy. The therapeutic strategy to reduce NLRP3 inflammasome activity can be separated in compounds that affect NLRP3 inflammasome assembly and activation, caspase- 1 activation, and IL-1 or IL18 pathways. $^{77}$

Interference with the IL-1 signaling pathway is the beststudied therapy to ameliorate NLRP3 activation because it is an approved therapy for autoinflammatory syndromes, including CAPS syndromes. Anti-IL-1 therapies are based on a recombinant IL-1 receptor antagonist (anakinra), humanized monoclonal anti-IL-1 $\beta$ antibodies (canakinumab), or an IL-1 trap produced by recombinant IL-1R (rilonacept). IL-1 receptor antagonist ameliorates inflammasome-dependent ASH in mice. ${ }^{78}$ Based on these results, a phase 2 study was initiated to test the efficacy of anakinra (plus pentoxifylline and zinc sulfate) compared with methylprednisolone in severe acute alcoholic hepatitis. ${ }^{79}$ Anakinra administration in various models of liver fibrosis ${ }^{80-82}$ also resulted in an ameliorated hepatocellular damage and deposition of matrix components, and a substantial decrease of HSC activation and serum levels of fibrosis markers, such as procollagen III $\mathrm{N}$-terminal propeptide. However, liver fibrosis was only partially reverted by administration of anakinra in other murine models, such as BDL-induced liver fibrosis ${ }^{61}$ and global hyperactivation of inflammasome. ${ }^{54}$

Programmed cell death, the hallmark of NASH that contributes to liver injury and fibrosis, is executed by numerous caspases. As outlined above, several studies provide strong evidence that treatment with pan-caspase inhibitors reduce liver injury and fibrosis in murine models of NASH as well as in patients. ${ }^{83-85}$ 
P2X7 belongs to purinergic receptor family that is activated by high concentrations of extracellular ATP, and its activation is a strong trigger of the NLRP3 inflammasome. ${ }^{7,86,87}$ The administration of a specific inhibitor of P2X7 (A438079) to mice in a $\mathrm{CCl}_{4}$-induced liver fibrosis, substantially attenuated hepatocyte death and inflammatory infiltration, and showed a reduced expression of proinflammatory cytokines and activation of fibrogenic factors that become apparent in a reduced deposition of collagen in the liver. ${ }^{88}$ The ligands of all these sensors are also increased, including ATP, HMGB1, uric acid, or lipopolysaccharide (LPS), in NASH and ASH models ${ }^{89,90}$ and their depletion attenuate the progression of the liver diseases and inflammasome activation and the secretion of its major downstream cytokine, IL- $1 \beta .{ }^{90}$ To date, only very few drugs have been described targeting the inflammasome directly and no study has evaluated the applicability and efficacy of an inflammasome blockade in models of NASH. Pfizer was pioneering developing IL-1 $\beta$-release inhibitory drugs and developed CRID3-a member of the class of diarylsulfonylurea containing compounds called cytokine release inhibitory drugs (CRIDs). ${ }^{48,91}$ Recently, CRID3 was renamed to MCC950, and was found to be a potent, selective, smallmolecule inhibitor of NLRP3 inflammasome activation. MCC950 blocked canonical and noncanonical NLRP3 activation at nanomolar concentrations. ${ }^{92}$ MCC950 specifically inhibited activation of NLRP3, but not the AIM2, NLRC4, or NLRP1 inflammasomes. MCC950 reduced IL-1 $\beta$ production in vivo and attenuated the severity of experimental autoimmune encephalomyelitis, a disease model of multiple sclerosis, and models of dermal and pulmonary inflammation. ${ }^{92,93}$ Furthermore, MCC950 treatment rescued neonatal lethality in a mouse model of CAPS and was active in ex vivo samples from individuals with Muckle-Wells syndrome. ${ }^{92}$ A recent study from our group using two murine models of NASH suggested that MCC950 is a potential novel therapy for fibrosis associated with this disease. ${ }^{94}$ Future studies are warranted to decipher the role of targeting cytosolic receptors as antifibrotic treatment.

\section{Future Perspectives}

In summary, in recent years we have gained significant insights into the central role of inflammation and cell death during the development of liver fibrosis. Inflammasomes are key modulators and translators of these processes when the liver is exposed to a variety of danger signals inducing the activation of proinflammatory caspase 1 , and the release of IL-1 $\beta$ and IL-18, as well as trigger pyroptotic cell death. These processes can initiate and perpetuate an abnormal woundhealing response with the principle cellular target being the activation of HSCs. Although a basic understanding of HSC activation now exists, precise biological mechanisms for translational and therapeutic benefit are unclear. As a result, effective treatments to prevent or reverse hepatic fibrosis have not yet been developed. The growing evidence from various studies that have uncovered a novel role of the NLRP3 inflammasome as a modulator of HSC biology and fibrosis suggest this pathway may represent an attractive target for antifibrotic therapies. In addition, they point at exciting new areas to further explore basic molecular and cellular mechanisms involved in fibrogenesis and liver fibrosis development.

\begin{tabular}{|c|c|}
\hline Abbreviations & \\
\hline a-SMA & $\alpha$ smooth muscle \\
\hline ASC & $\begin{array}{l}\text { adaptor protein apoptosis speck-like } \\
\text { protein }\end{array}$ \\
\hline ASH & alcoholic steatohepatitis \\
\hline ATP & adenosine triphosphate \\
\hline BDL & bile duct ligation \\
\hline CAPS & $\begin{array}{l}\text { cryopyrin-associated periodic } \\
\text { syndromes }\end{array}$ \\
\hline CARD & $\begin{array}{l}\text { caspase activation and recruitment } \\
\text { domain }\end{array}$ \\
\hline CDAA & choline-deficient amino acid-defined \\
\hline CreLT & lecithin retinol acyltransferase Cre \\
\hline CRIDs & cytokine release inhibitory drugs \\
\hline DAMPs & $\begin{array}{l}\text { damage-associated molecular } \\
\text { patterns }\end{array}$ \\
\hline $\mathrm{HCV}$ & hepatitis C virus \\
\hline HMGB1 & high-mobility group box 1 \\
\hline HSC & hepatic stellate cell \\
\hline IL & interleukin \\
\hline IL-1 receptor type I & IL-1 receptor type I \\
\hline NASH & nonalcoholic steatohepatitis \\
\hline NF-кB & $\begin{array}{l}\text { nuclear factor kappa-light-chain-en- } \\
\text { hancer of activated B cells }\end{array}$ \\
\hline NLRs & $\begin{array}{l}\text { nucleotide-binding domain and leu- } \\
\text { cine-rich repeat containing receptors }\end{array}$ \\
\hline PYD & pyrin domain \\
\hline ROS & reactive oxygen species \\
\hline TGF- $\beta$ & transforming growth factor- $\beta$ \\
\hline TLR & Toll-like receptor. \\
\hline
\end{tabular}

\section{References}

1 Byass P. The global burden of liver disease: a challenge for methods and for public health. BMC Med 2014;12:159

2 Schuppan D, Kim YO. Evolving therapies for liver fibrosis. J Clin Invest 2013;123(5):1887-1901

3 Charlton MR, Burns JM, Pedersen RA, Watt KD, Heimbach JK, Dierkhising RA. Frequency and outcomes of liver transplantation for nonalcoholic steatohepatitis in the United States. Gastroenterology 2011;141(4):1249-1253

4 Darwish Murad S, Metselaar HJ. The invasion of fatty liver disease in liver transplantation. Transpl Int 2016;29(4):416-417

5 Xu J, Liu X, Koyama Y, et al. The types of hepatic myofibroblasts contributing to liver fibrosis of different etiologies. Front Pharmacol 2014;5:167 
6 Mederacke I, Hsu CC, Troeger JS, et al. Fate tracing reveals hepatic stellate cells as dominant contributors to liver fibrosis independent of its aetiology. Nat Commun 2013;4:2823

7 Wree A, Mehal WZ, Feldstein AE. Targeting cell death and sterile inflammation loop for the treatment of nonalcoholic steatohepatitis. Semin Liver Dis 2016;36(1):27-36

8 Thapaliya S, Wree A, Povero D, et al. Caspase 3 inactivation protects against hepatic cell death and ameliorates fibrogenesis in a diet-induced NASH model. Dig Dis Sci 2014;59(6):11971206

9 Luheshi NM, Giles JA, Lopez-Castejon G, Brough D. Sphingosine regulates the NLRP3-inflammasome and IL- $1 \beta$ release from macrophages. Eur J Immunol 2012;42(3):716-725

10 Takehara T, Tatsumi T, Suzuki T, et al. Hepatocyte-specific disruption of Bcl-xL leads to continuous hepatocyte apoptosis and liver fibrotic responses. Gastroenterology 2004;127(4):1189-1197

11 Zhan S-S, Jiang JX, Wu J, et al. Phagocytosis of apoptotic bodies by hepatic stellate cells induces NADPH oxidase and is associated with liver fibrosis in vivo. Hepatology 2006;43(3):435-443

12 Garcia-Martinez I, Santoro N, Chen Y, et al. Hepatocyte mitochondrial DNA drives nonalcoholic steatohepatitis by activation of TLR9. J Clin Invest 2016;126(3):859-864

13 Povero D, Panera N, Eguchi A, et al. Lipid-induced hepatocytederived extracellular vesicles regulate hepatic stellate cell via microRNAs targeting PPAR- $\gamma$. Cell Mol Gastroenterol Hepatol 2015;1(6):646-663.e4

14 Ting JP, Lovering RC, Alnemri ES, et al. The NLR gene family: a standard nomenclature. Immunity 2008;28(3):285-287

15 Latz E, Xiao TS, Stutz A. Activation and regulation of the inflammasomes. Nat Rev Immunol 2013;13(6):397-411

16 Pelegrin PInflammasome activation by danger signals In: Couillin I, Petrilli V, Martinon F, eds. The Inflammasomes. Basel, Switzerland: Springer Basel; 2011:101-121

17 Masumoto J, Taniguchi S, Ayukawa K, et al. ASC, a novel 22-kDa protein, aggregates during apoptosis of human promyelocytic leukemia HL-60 cells. J Biol Chem 1999;274(48):33835-33838

18 Fernandes-Alnemri T, Wu J, Yu JW, et al. The pyroptosome: a supramolecular assembly of ASC dimers mediating inflammatory cell death via caspase-1 activation. Cell Death Differ 2007;14(9): 1590-1604

19 Lu A, Magupalli VG, Ruan J, et al. Unified polymerization mechanism for the assembly of ASC-dependent inflammasomes. Cell 2014;156(6):1193-1206

20 Murakami T, Ockinger J, Yu J, et al. Critical role for calcium mobilization in activation of the NLRP3 inflammasome. Proc Natl Acad Sci U S A 2012;109(28):11282-11287

21 Hornung V, Bauernfeind F, Halle A, et al. Silica crystals and aluminum salts activate the NALP3 inflammasome through phagosomal destabilization. Nat Immunol 2008;9(8):847-856

22 Zhou R, Yazdi AS, Menu P, Tschopp J. A role for mitochondria in NLRP3 inflammasome activation. Nature 2011;469(7329):221-225

23 Katsnelson MA, Rucker LG, Russo HM, Dubyak GRK. K+ efflux agonists induce NLRP3 inflammasome activation independently of Ca2+ signaling. J Immunol 2015;194(8):3937-3952

24 Muñoz-Planillo R, Kuffa P, Martínez-Colón G, Smith BL, Rajendiran TM, Núñez G. $\mathrm{K}^{+}$efflux is the common trigger of NLRP3 inflammasome activation by bacterial toxins and particulate matter. Immunity 2013;38(6):1142-1153

25 Pétrilli V, Papin S, Dostert C, Mayor A, Martinon F, Tschopp J. Activation of the NALP3 inflammasome is triggered by low intracellular potassium concentration. Cell Death Differ 2007; 14(9):1583-1589

26 Mariathasan S, Weiss DS, Newton K, et al. Cryopyrin activates the inflammasome in response to toxins and ATP. Nature 2006; 440(7081):228-232

27 Compan V, Baroja-Mazo A, López-Castejón G, et al. Cell volume regulation modulates NLRP3 inflammasome activation. Immunity $2012 ; 37(3): 487-500$
28 Groß CJ, Mishra R, Schneider KS, et al. K(+) efflux-independent NLRP3 inflammasome activation by small molecules targeting mitochondria. Immunity 2016;45(4):761-773

29 Dinarello CA. Immunological and inflammatory functions of the interleukin-1 family. Annu Rev Immunol 2009;27:519-550

30 Auron PE, Webb AC, Rosenwasser LJ, et al. Nucleotide sequence of human monocyte interleukin 1 precursor cDNA. Proc Natl Acad Sci U S A 1984;81(24):7907-7911

31 López-Castejón G, Brough D. Understanding the mechanism of IL-1 $\beta$ secretion. Cytokine Growth Factor Rev 2011;22(4):189-195

32 Fantuzzi G, Ku G, Harding MW, et al. Response to local inflammation of IL-1 beta-converting enzyme- deficient mice. J Immunol 1997;158(4):1818-1824

33 MacKenzie A, Wilson HL, Kiss-Toth E, Dower SK, North RA, Surprenant A. Rapid secretion of interleukin-1beta by microvesicle shedding. Immunity 2001;15(5):825-835

34 Cullen SP, Kearney CJ, Clancy DM, Martin SJ. Diverse activators of the NLRP3 inflammasome promote il- $1 \beta$ secretion by triggering necrosis. Cell Reports 2015;11(10):1535-1548

35 Liu X, Zhang Z, Ruan J, et al. Inflammasome-activated gasdermin D causes pyroptosis by forming membrane pores. Nature 2016; 535(7610):153-158

36 Martín-Sánchez F, Diamond C, Zeitler M, et al. Inflammasomedependent IL-1 $\beta$ release depends upon membrane permeabilisation. Cell Death Differ 2016;23(7):1219-1231

37 de Torre-Minguela C, Barberà-Cremades M, Gómez AI, MartínSánchez F, Pelegrín P. Macrophage activation and polarization modify P2X7 receptor secretome influencing the inflammatory process. Sci Rep 2016;6:22586

38 Keller M, Rüegg A, Werner S, Beer H-D. Active caspase- 1 is a regulator of unconventional protein secretion. Cell 2008;132(5): 818-831

39 Gross O, Yazdi AS, Thomas CJ, et al. Inflammasome activators induce interleukin- $1 \alpha$ secretion via distinct pathways with differential requirement for the protease function of caspase- 1 . Immunity 2012;36(3):388-400

40 Yang D, Postnikov YV, Li Y, et al. High-mobility group nucleosomebinding protein 1 acts as an alarmin and is critical for lipopolysaccharide-induced immune responses. J Exp Med 2012;209(1):157-171

41 Miao EA, Rajan JV, Aderem A. Caspase-1-induced pyroptotic cell death. Immunol Rev 2011;243(1):206-214

42 Kayagaki N, Warming S, Lamkanfi M, et al. Non-canonical inflammasome activation targets caspase-11. Nature 2011; 479(7371):117-121

43 ShiJ, Zhao Y, Wang Y, et al. Inflammatory caspases are innate immune receptors for intracellular LPS. Nature 2014;514(7521):187-192

44 Kayagaki N, Stowe IB, Lee BL, et al. Caspase-11 cleaves gasdermin D for non-canonical inflammasome signalling. Nature 2015; 526(7575):666-671

45 Shi J, Zhao Y, Wang K, et al. Cleavage of GSDMD by inflammatory caspases determines pyroptotic cell death. Nature 2015; 526(7575):660-665

46 Sborgi L, Rühl S, Mulvihill E, et al. GSDMD membrane pore formation constitutes the mechanism of pyroptotic cell death. EMBO J 2016;35(16):1766-1778

47 Ding J, Wang K, Liu W, et al. Pore-forming activity and structural autoinhibition of the gasdermin family. Nature 2016;535(7610): $111-116$

48 Perregaux DG, McNiff P, Laliberte R, et al. Identification and characterization of a novel class of interleukin-1 post-translational processing inhibitors. J Pharmacol Exp Ther 2001;299(1):187-197

49 Baroja-Mazo A, Martín-Sánchez F, Gomez AI, et al. The NLRP3 inflammasome is released as a particulate danger signal that amplifies the inflammatory response. Nat Immunol 2014;15(8): 738-748

50 Franklin BS, Bossaller L, De Nardo D, et al. The adaptor ASC has extracellular and 'prionoid' activities that propagate inflammation. Nat Immunol 2014;15(8):727-737 
51 Sagoo P, Garcia Z, Breart B, et al. In vivo imaging of inflammasome activation reveals a subcapsular macrophage burst response that mobilizes innate and adaptive immunity. Nat Med 2016;22(1):64-71

52 Tzeng T-CJ, Schattgen S, Monks B, et al. A fluorescent reporter mouse for inflammasome assembly demonstrates an important role for cell-bound and free ASC specks during in vivo infection. Cell Reports 2016;16(2):571-582

53 Boaru SG, Borkham-Kamphorst E, Tihaa L, Haas U, Weiskirchen R. Expression analysis of inflammasomes in experimental models of inflammatory and fibrotic liver disease. J Inflamm (Lond) 2012; $9(1): 49$

54 Wree A, Eguchi A, McGeough MD, et al. NLRP3 inflammasome activation results in hepatocyte pyroptosis, liver inflammation, and fibrosis in mice. Hepatology 2014;59(3):898-910

55 Wree A, McGeough MD, Peña CA, et al. NLRP3 inflammasome activation is required for fibrosis development in NAFLD. J Mol Med (Berl) 2014;92(10):1069-1082

56 Isoda K, Sawada S, Ayaori M, et al. Deficiency of interleukin-1 receptor antagonist deteriorates fatty liver and cholesterol metabolism in hypercholesterolemic mice. J Biol Chem 2005;280(8): 7002-7009

57 Kolb M, Margetts PJ, Anthony DC, Pitossi F, Gauldie J. Transient expression of IL-1beta induces acute lung injury and chronic repair leading to pulmonary fibrosis. J Clin Invest 2001;107(12):15291536

58 Gasse P, Mary C, Guenon I, et al. IL-1R1/MyD88 signaling and the inflammasome are essential in pulmonary inflammation and fibrosis in mice. J Clin Invest 2007;117(12):3786-3799

59 Ludwig-Portugall I, Bartok E, Dhana E, et al. An NLRP3-specific inflammasome inhibitor attenuates crystal-induced kidney fibrosis in mice. Kidney Int 2016;90(3):525-539

60 Yaping Z, Ying W, Luqin D, Ning T, Xuemei A, Xixian Y. Mechanism of interleukin-1 $\beta$-induced proliferation in rat hepatic stellate cells from different levels of signal transduction. APMIS 2014; 122(5):392-398

61 Reiter FP, Wimmer R, Wottke L, et al. Role of interleukin-1 and its antagonism of hepatic stellate cell proliferation and liver fibrosis in the Abcb4(-/-) mouse model. World J Hepatol 2016;8(8): 401-410

62 Yan C, Zhou L, Han Y-P. Contribution of hepatic stellate cells and matrix metalloproteinase 9 in acute liver failure. Liver Int 2008; 28(7):959-971

63 Tang N, Zhang YP, Ying W, Yao XX. Interleukin-1 $\beta$ upregulates matrix metalloproteinase-13 gene expression via c-Jun N-terminal kinase and p38 MAPK pathways in rat hepatic stellate cells. Mol Med Rep 2013;8(6):1861-1865

64 Miura K, Kodama Y, Inokuchi S, et al. Toll-like receptor 9 promotes steatohepatitis by induction of interleukin-1beta in mice. Gastroenterology 2010;139(1):323-34.e7

65 Kamari Y, Shaish A, Vax E, et al. Lack of interleukin- $1 \alpha$ or interleukin- $1 \beta$ inhibits transformation of steatosis to steatohepatitis and liver fibrosis in hypercholesterolemic mice. J Hepatol 2011;55(5):1086-1094

66 Salguero Palacios R, Roderfeld M, Hemmann S, et al. Activation of hepatic stellate cells is associated with cytokine expression in thioacetamide-induced hepatic fibrosis in mice. Lab Invest 2008; 88(11):1192-1203

67 Gieling RG, Wallace K, Han YP. Interleukin-1 participates in the progression from liver injury to fibrosis. Am J Physiol Gastrointest Liver Physiol 2009;296(6):G1324-G1331

68 Dixon LJ, Flask CA, Papouchado BG, Feldstein AE, Nagy LE. Caspase- 1 as a central regulator of high fat diet-induced nonalcoholic steatohepatitis. PLoS One 2013;8(2):e56100

69 Dixon LJ, Berk M, Thapaliya S, Papouchado BG, Feldstein AE. Caspase-1-mediated regulation of fibrogenesis in diet-induced steatohepatitis. Lab Invest 2012;92(5):713-723

70 Miura K, Yang L, van Rooijen N, Brenner DA, Ohnishi H, Seki E. Tolllike receptor 2 and palmitic acid cooperatively contribute to the development of nonalcoholic steatohepatitis through inflammasome activation in mice. Hepatology 2013;57(2):577-589

71 Watanabe A, Sohail MA, Gomes DA, et al. Inflammasome-mediated regulation of hepatic stellate cells. Am J Physiol Gastrointest Liver Physiol 2009;296(6):G1248-G1257

72 Johnson C, Wree A, McGeough MD, et al. Hepatic stellate cell specific NLRP3 inflammasome activation results in spontaneous fibrosis. Hepatology 2016;64:100A

73 Negash AA, Ramos HJ, Crochet N, et al. IL-1 $1 \beta$ production through the NLRP3 inflammasome by hepatic macrophages links hepatitis $C$ virus infection with liver inflammation and disease. PLoS Pathog 2013;9(4):e1003330

74 Wree A, Schlattjan M, Bechmann LP, et al. Adipocyte cell size, free fatty acids and apolipoproteins are associated with non-alcoholic liver injury progression in severely obese patients. Metabolism 2014;63(12):1542-1552

75 Cai SM, Yang RQ Li Y, et al. Angiotensin-(1-7) improves liver fibrosis by regulating the NLRP3 inflammasome via redox balance modulation. Antioxid Redox Signal 2016;24(14):795-812

76 Adams LA, Wree A, Melton P, et al. Serum marker of inflammasome activity correlates with liver injury in nonalcoholic fatty liver disease and is influenced by genetic polymorphisms. Hepatology 2015;62:1273A

77 López-Castejón G, Pelegrín P. Current status of inflammasome blockers as anti-inflammatory drugs. Expert Opin Investig Drugs 2012;21(7):995-1007

78 Petrasek J, Bala S, Csak T, et al. IL-1 receptor antagonist ameliorates inflammasome-dependent alcoholic steatohepatitis in mice. J Clin Invest 2012;122(10):3476-3489

79 Mitchell MA phase 2, efficacy study of anakinra, pentoxifylline, and zinc compared to methylprednisolone in severe acute alcoholic hepatitis 2016. Available at: https://clinicaltrials.gov/ct2/ show/NCT01809132

80 Mancini R, Benedetti A, Jezequel A-M. An interleukin-1 receptor antagonist decreases fibrosis induced by dimethylnitrosamine in rat liver. Virchows Arch 1994;424(1):25-31

81 Zhu R-Z, Xiang D, Xie C, et al. Protective effect of recombinant human IL-1Ra on CCl4-induced acute liver injury in mice. World J Gastroenterol 2010;16(22):2771-2779

82 Lodder J, Denaës T, Chobert MN, et al. Macrophage autophagy protects against liver fibrosis in mice. Autophagy 2015;11(8): 1280-1292

83 Witek RP, Stone WC, Karaca FG, et al. Pan-caspase inhibitor VX166 reduces fibrosis in an animal model of nonalcoholic steatohepatitis. Hepatology 2009;50(5):1421-1430

84 Anstee QM, Concas D, Kudo H, et al. Impact of pan-caspase inhibition in animal models of established steatosis and nonalcoholic steatohepatitis. J Hepatol 2010;53(3):542-550

85 Barreyro FJ, Holod S, Finocchietto PV, et al. The pan-caspase inhibitor Emricasan (IDN-6556) decreases liver injury and fibrosis in a murine model of non-alcoholic steatohepatitis. Liver Int 2015;35(3):953-966

86 Szabo G, Iracheta-Vellve A. Inflammasome activation in the liver: focus on alcoholic and non-alcoholic steatohepatitis. Clin Res Hepatol Gastroenterol 2015;39(Suppl 1):S18-S23

87 Szabo G, Csak T. Inflammasomes in liver diseases. J Hepatol 2012; 57(3):642-654

88 Huang C, Yu W, Cui H, et al. P2X7 blockade attenuates mouse liver fibrosis. Mol Med Rep 2014;9(1):57-62

89 Ganz M, Bukong TN, Csak T, et al. Progression of non-alcoholic steatosis to steatohepatitis and fibrosis parallels cumulative accumulation of danger signals that promote inflammation and liver tumors in a high fat-cholesterol-sugar diet model in mice. J Transl Med 2015;13:193

90 Iracheta-Vellve A, Petrasek J, Satishchandran A, et al. Inhibition of sterile danger signals, uric acid and ATP, prevents inflammasome activation and protects from alcoholic steatohepatitis in mice. J Hepatol 2015;63(5):1147-1155 
91 Laliberte RE, Perregaux DG, Hoth LR, et al. Glutathione s-transferase omega 1-1 is a target of cytokine release inhibitory drugs and may be responsible for their effect on interleukin1beta posttranslational processing. J Biol Chem 2003;278(19): 16567-16578

92 Coll RC, Robertson AAB, Chae JJ, et al. A small-molecule inhibitor of the NLRP3 inflammasome for the treatment of inflammatory diseases. Nat Med 2015;21(3):248-255
93 Primiano MJ, Lefker BA, Bowman MR, et al. Efficacy and pharmacology of the NLRP3 Inflammasome inhibitor CP-456,773 (CRID3) in murine models of dermal and pulmonary inflammation. J Immunol 2016;197(6):2421-2433

94 Mridha AR, Wree A, Robertson AA, et al. NLRP3 inflammasome blockade reduces liver inflammation and fibrosis in experimental NASH in mice. J Hepatol 2017; pii:S0168-8278 (17)30056-30059. doi: 10.1016/j.jhep.2017.01.022. [Epub ahead of print] 